\title{
Clinical pharmacists' scope of knowledge for medication therapy management in chronic kidney disease patients
}

\author{
Sirimalla Shivaprasad ${ }^{1}(\mathbb{D})$, Uday Venkat Mateti ${ }^{1}$, Pradeep Shenoy $^{2}$ (D) Chakrakodi Shashidhara Shastry $^{1}$, \\ Sreedhar Dharmagadda ${ }^{3}$ (I) \\ ${ }^{1}$ NGSM Institute of Pharmaceutical Sciences, Nitte Deemed to be University, Deralakatte, Mangaluru, Karnataka, India \\ 2 KS Hegde Medical Academy, Nitte Deemed to be University, Deralakatte, Mangaluru, Karnataka, India \\ ${ }^{3}$ Manipal College of Pharmaceutical Sciences, Manipal Academy of Higher Education, Manipal, Karnataka, India
}

\section{Keywords}

Chronic kidney disease

Medication therapy management

Medication therapy review

Quality of life

\section{Correspondence}

Uday Venkat Mateti

Department of Pharmacy Practice

NGSM Institute of Pharmaceutical

Sciences

Deralakatte, Mangaluru

Karnataka, India 575018

udayvenkatmateti@gmail.com

\begin{abstract}
Medication therapy management (MTM) was first implemented and introduced for chronically ill patients and those taking multiple prescription drugs. The MTM has five steps, namely medication therapy review (MTR), personal medication record (PMR), medication-related action plan (MAP), intervention or referral, and finally, documentation. After receiving MTM services, patients will gain knowledge on medicines, which may decrease non-adherence to treatment and increase its efficacy. Studies have shown the positive impact of MTM on geriatric, pediatric, and chronically ill patients and those on polypharmacy. MTM services may improve medication adherence, decrease healthcare costs, and improve the quality of life (QoL) of patients with chronic kidney disease (CKD) by addressing various issues like anaemia, metabolic acidosis, protein management, fluid management, electrolyte management, dosage adjustment based on eGFR, vaccination, and medication-related problems and intervening with the education about the disease, drugs, and lifestyle modifications.
\end{abstract}

\section{Introduction}

The concept of medication therapy management (MTM) was first presented in the "Medicare Prescription Drug Improvement and Modernization Act of 2003" by federal legislation in the United States. It was primarily introduced in patients with chronic illnesses and those taking multiple prescription drugs (Public law 108-173, 2003). The pharmacist is responsible for the provision of MTM services, which include five fundamental components: medication therapy review (MTR), personal medication record (PMR), medication-related action plan (MAP), referral or intervention, and finally, the documentation of activities. Studies in Western countries have shown the positive effect of MTM on pediatric and geriatric populations, especially in patients with chronic illnesses and those taking multiple medications (Lin et al., 2018).

In India, only a few studies were conducted on MTM. However, MTM services are established in developed countries for chronic diseases like diabetes, hypertension, cancer, and CKD. It was identified that insufficient and low-quality data documents a clinical pharmacist's intervention among CKD patients (Al Raiisi $\mathrm{F}$ et al., 2019). Similar studies that have been done earlier have primarily focused on a few issues, such as the management of anaemia, medication-related problems, and dose adjustments (Ohnishi J, 2011; Mason, 2011). The clinical pharmacist can focus a wide range of issues on addressing CKD patients, such as dose adjustment in CKD, protein management, anaemia management, phosphate balance, vaccination, fluid 
balance, corrected calcium, potassium management, metabolic acidosis, sodium management, MRPs, and effectively handling these concerns can improve the clinical, economic, and humanistic outcomes of patients with CKD.

CKD is characterised by continuous, permanent kidney impairment, with an estimated glomerular filtration rate (eGFR) of below $60 \mathrm{~mL}$ per minute or evidence of kidney damage or excretion of protein and blood in the urine for at least three months (Applied therapeutics the clinical use of drugs, 2013). Non-communicable diseases, such as CKD, are a leading source of morbidity and mortality (Couser et al., 2011). There were 697.5 million cases of all-stage CKD in 2017, representing a worldwide prevalence of $9.1 \%$ (8.5 to 9.8) (GBD Chronic Kidney Disease Collaboration, 2020). In India, the prevalence of CKD was $17.2 \%$ (Singh et al., 2013), and the most prevailing risks and characteristics for CKD were hypertension, anaemia, and diabetes (Stemer \& Lemmens-Gruber, 2011). Diabetes and hypertension are the foremost cause of CKD. Optimal blood sugar levels and blood pressure (BP) control are required to delay its progression and reduce morbidity and mortality (Applied therapeutics the clinical use of drugs, 2013). Treatment of CKD-related anaemia is paramount for decreasing cardiovascular disease (CVD) complications. Progression of CKD from starting to the late stage can lead to new symptoms and associated complications, including anaemia, hypertension, renal osteodystrophy, hyperlipidaemia, metabolic acidosis, electrolyte abnormalities, and other comorbidities involving malnourishment, pruritus, and uremic bleeding (Stemer \& Lemmens-Gruber, 2011). Drugrelated morbidity and medication-related problems (MRPs) may be exacerbated by using multiple medications, non-adherence to treatment, and frequent dosage changes. Pharmacists need timely interventions to prevent further progression of CKD. MTM services provided by the clinical pharmacist significantly contribute to a multidisciplinary team, providing safe, effective, and financial care. Clinical pharmacists are pharmacotherapy experts working with other healthcare providers regularly to meet multiple and often unresolved demands for pharmacotherapy optimization.

The purpose of this review is to assess the role of the pharmacist in CKD management through the provision of MTM services and highlight areas of focus, such as management of anaemia in renal dose adjustment, BP management, $\mathrm{Na}^{+}$and water balance, phosphorous and calcium balance, and management of proteinuria in CKD patients.

The clinical pharmacist should focus on the following areas.

\section{Dose selection and adjustment}

Drug excretion is usually impaired in patients with CKD. The pharmacokinetic parameters of patients with kidney disease differ from those of patients with normal kidney function. In these people, drug selection and dosage is crucial to prevent undesirable drug effects. Attempts to minimise dosage errors can lower the rate of adverse effects, decrease costs, and enhance overall healthcare delivery (Hassan, AlRamahi, Aziz, \& Ghazali, 2009).

Generally, the Cockcroft-Gault formula will be used to calculate creatinine clearance $(\mathrm{CrCl})$.

$$
\begin{aligned}
& \text { In males, } \quad \mathrm{CrCl}=\frac{(140-\text { age }) \times \text { weight }}{72 \times \text { Serum Creatinine }(\mathrm{SrCr})} \\
& \text { In females, } \mathrm{CrCl}=\frac{(140-\text { age }) \times \text { weight }}{72 \times \mathrm{SrCr}} \times 0.85
\end{aligned}
$$

In children, the Schwartz formula will be used to estimate the glomerular filtration rate (eGFR), mainly relying on the child's height since height correlates with muscle mass.

$$
\text { eGFR }=0.55 \times \frac{\text { Height }}{\mathrm{SrCr}}
$$

Some medications should be avoided in CKD patients with an eGFR<60 $\mathrm{mL} / \mathrm{min} / 1.73 \mathrm{~m}^{2}$, including nitrofurantoin and phenazopyridine (Onder et al., 2006; MEDSAFE New Zealand Medicines and Medical Devices Safety Authority, 2015). If eGFR $<30$ $\mathrm{mL} / \mathrm{min} / 1.73 \mathrm{~m}^{2}$, then the risk is increased, and gadolinium must be avoided as much as possible. This chemical contrast agent used in magnetic resonance imaging has been linked to nephrogenic systemic fibrosis in certain patients.

Adverse drug events (ADEs) occur when drugs are used inadequately, which can increase hospital stays, overburdening, and healthcare system expenditure. Most ADEs are unpreventable, but some are caused by management errors and may be preventable. Errors in dosing are the leading cause of medication-related problems (MRPs) in CKD patients. Therapeutic efficacy increases with proper dosage, decreased toxicity, and reduced workload. It also has a financial impact, eliminating the costs associated with saving on MRPs and drug costs (Hassan et al., 2009). Some drugs need dose adjustment as per the GFR to prevent further progression of CKD. The below information contains the dose adjustment according to the GFR range (Michigan Medicine University of Michigan, 2019).

\section{Dosage adjustment in hypertension}

Most CKD patients suffer from hypertension, which is both a cause and a consequence of the disease. 
Hypertension control is crucial in people with CKD because it slows disease progression and lowers the risk of heart disease. Controlling hypertension will reduce renal disease progression and CVD morbidity and mortality. According to the JNC-8 guidelines, the target BP and treatment threshold are the same for those over age 18 years with hypertension along with CKD or diabetes mellitus. For hypertension, JNC-8 guidelines suggested a goal BP of less than 140/90 $\mathrm{mmHg}$. In people with CKD 18 years or older, early (or add-on) antihypertensive therapy may require ACEI or ARB to increase renal outcomes, applicable for all hypertensive CKD patients, independent of race or diabetes status (James et al., 2014). In CKD patients, most drugs are not eliminated adequately from the body. Hence, dose adjustment is needed for hypertension patients with CKD based on the GFR to avoid ADRs and ADEs. Medications requiring adjustment based on GFR include angiotensinconverting enzyme inhibitors (enalapril, captopril, benazepril, and ramipril), aldosterone antagonists (eplerenone and spironolactone), potassium-sparing diuretics (amiloride), and beta-blockers (atenolol) (Michigan Medicine University of Michigan, 2019).

\section{Dosage adjustment in diabetes in patients with CKD}

Dose adjustment is needed to manage diabetes mellitus in CKD patients to avoid toxicity or adverse events caused by antidiabetic drugs. Some of the antidiabetic drugs, which require dose adjustments based on GFR are biguanide (metformin), sulfonylureas (glipizide and glyburide), thiazolidinediones (pioglitazone), dipeptidyl peptidase-4 (DPP-4) inhibitors (sitagliptin, saxagliptin, and alogliptin), and incretin mimetics (exenatide). Some of the drugs that should be avoided completely as per GFR are metformin (should be avoided completely for people with GFR less than $30 \mathrm{~mL} / \mathrm{min} / 1.73 \mathrm{~m}^{2}$ since it increases the likelihood of lactic acidosis). The risk vs benefit ratio should be assessed if the physician wants to give metformin for patients with GFR less than 30 $\mathrm{mL} / \mathrm{min} / 1.73 \mathrm{~m}^{2}$. Glyburide should be avoided in CKD patients if GFR is less than $30 \mathrm{~mL} / \mathrm{min} / 1.73 \mathrm{~m}^{2}$ since it accumulates in the body, causing prolonged hypoglycemia. Pioglitazone should be cautiously used because it can cause dose-related oedema. Incretin mimetics like exenatide should be avoided in CKD patients because it can cause acute renal failure and worsen the chronic renal condition, as reported by post-marketing studies (Michigan Medicine University of Michigan, 2019).

\section{Anaemia in CKD patients}

Anaemia is the most common complication that develops in CKD patients and is associated with emergencies, including hospitalisation, cardiovascular disease, and death. The target $\mathrm{Hb}$ level should be 11 to $12 \mathrm{~g} / \mathrm{dL}$ in CKD patients not on dialysis (Applied therapeutics the clinical use of drugs, 2013; Mimura, Tanaka, Nangaku, 2015). Generally, anaemia develops due to erythropoietin deficiency in patients with CKD. The administration of erythropoiesis-stimulating agents (ESAs) will increase the production of red blood cells and decrease the need for blood transfusions. However, ESAs add risks to CKD patients, including increased BP and thrombotic issues; therefore, ESAs should be given at the lowest possible dose. Before administering treatment for anaemia, physicians should check the potential factors that cause anaemia, including iron deficiency anaemia. In this condition, supplementation with iron is needed before starting treatment with ESAs (Rogers, Leung, Beaulieu, Levin, Burnett, \& Zienkiewicz, 2011).

\section{Protein management}

High protein intake leads to hyper-glomerular filtration and intra-glomerular pressure, which can damage the structure of the glomeruli and the kidneys further. As a result, a low protein intake of $0.6-0.8 \mathrm{~g} / \mathrm{kg}$ per day is indicated in CKD patients (Ko et al., 2017). Creatinine and urea are the byproducts of proteins. The kidneys generally remove these byproducts. More protein makes the kidneys work hard; therefore, CKD patients should eat less protein. Patients should avoid plantbased high protein foods such as peas, beans, dals, soy foods, soy milk, tofu, nuts, and nut spreads like almond butter, peanut butter, and soy nut butter. They should prefer plant-based fewer protein foods such as bread, chapati, oats, grits, grains, pasta, noodles, rice, and rice milk (Protein Tips for People with Chronic Kidney Disease, 2010).

\section{Phosphate balance}

Hyperphosphataemia is the condition that mainly occurs in CKD patients as kidneys cannot excrete enough phosphate in the urine, causing high phosphate levels in the serum that, higher than $4.5 \mathrm{mg} / \mathrm{dL}$, the normal levels being 3.5-4.5 mg/dL (Wojcicki, 2013). In the early stages of CKD, hyperphosphataemia is mainly controlled by limiting the phosphate contained foods. Foods low in phosphorous are vegetables and fruits, rice milk, bread, pasta, rice, corn, and rice cereals. Highphosphorus foods included poultry and fish meat, dairy products, beans, dals, nuts, bran cereals, oatmeal, colas and some bottled iced tea (Phosphorus Tips for People with Chronic Kidney Disease, 2010). If not controlled by 
diet, phosphate binding agents (calcium-containing or noncalcium-containing) can control hyperphosphataemia. These agents can reduce phosphate absorption from the intestine by producing insoluble complexes with phosphates present in the food. Phosphate-binding agents can give good results if taken along with food. In patients with hypercalcaemia, anatomical bone disease, or imaging evidence of vascular calcification, non-calcium-phosphate binders are commonly used. If calcium-containing binders are prescribed for patients with an eGFR less than $60 \mathrm{~mL} / \mathrm{min} / 1.73 \mathrm{~m}^{2}$, the total daily calcium intake should not exceed $2000 \mathrm{mg}$ (Phosphorus Tips for People with Chronic Kidney Disease, 2010). In adults, calcium acetate can be used as a first-line phosphate binder in addition to diet to manage serum phosphate. If individuals are unable to tolerate calcium acetate, calcium carbonate should be used instead. In second-line phosphate binder treatment for adults with fourth to fifth stage of CKD and who are not on dialysis, a non-calcium-based binder such as sucroferric oxyhydroxide is considered if calcium-based phosphate binders are not tolerated, or hypercalcaemia exists, or parathyroid hormone blood levels are low (Hyperphosphatemia in chronic kidney disease overview, 2020).

\section{Vaccination in CKD patients}

Infections are the main concern for CKD patients, and some infections may be prevented by vaccination. However, live vaccines should not be advised in immune-deficient patients owing to the danger of vaccine-induced infections, except for varicella and MMR (measles, mumps and rubella) vaccines. The vaccines recommended in CKD are hepatitis $B$, pneumococcal, hepatitis $A$, and MMR vaccines. Hepatitis $B$ is recommended for all CKD patients. Patients should take four doses as early after contracting the disease, and the dosing schedule should be $0,1,2$, and 6 months. Patients over the age of 20 should receive $40 \mathrm{mcg} / \mathrm{mL}$ or two $20 \mathrm{mcg} / \mathrm{mL}$, while in those under 20 years old, $10 \mathrm{mcg}$ of hepatitis B is recommended. Pneumococcal vaccine should be taken every year at a dose of $15 \mathrm{mcg}$ intramuscularly. A booster dose is not needed. Patients above 19 should receive a first dose of the vaccine. Hepatitis $A$ is recommended for CKD people above 17 years of age at a dosing schedule of 0,6 , and 12 months and a dose of $1440 \mathrm{U}$, intramuscularly with no booster dose. MMR vaccine is recommended for CKD patients over 18 years of age. Only one dose should be given subcutaneously at a dose of $0.5 \mathrm{~mL}$ (Indian journal of nephrology, 2016).

\section{Fluid intake}

The primary function of the kidneys is to balance fluid in the body. In CKD patients, kidneys do not excrete enough fluid, causing fluid retention in the body with detrimental effects on health, such as oedema, shortness of breath, and inflammation. Oedema can result from sodium retention and consequent water retention. CKD patients in stage 1 or 2 should take sufficient fluids or water, around 1.8 litres, to help the kidneys be hydrated and work well (American Kidney Fund, 2021). If patients are in the later stages of CKD (third, fourth, and fifth stage) and not on dialysis, they should limit fluid intake to around 1 to 2 litres per day, depending on current dietary intake, the extent of volume overload, and urine output. The liquid allowance involves liquids taken in any form, including drinks, sauces, medicine, and fruits. The fluid limit is difficult to maintain in the later stages of CKD. Placing ice cubes in the mouth can relieve unpleasant dry mouth, but the patient should not be encouraged to swallow water (Clinical pharmacy and therapeutics, 2012).

\section{Metabolic acidosis}

Generally, the production of bicarbonates and excretion of acids $\left(\mathrm{H}^{+}\right)$are impaired in CKD patients and lead to metabolic acidosis. It contributes to several problems, including bone disease, abnormal protein metabolism, skeletal muscle loss and gradual GFR loss. Generally, metabolic acidosis is treated with a sodiumbased alkali like sodium bicarbonate $\left(\mathrm{NaHCO}_{3}\right)$ or sodium citrate instead of potassium-based alkali because potassium excretion in some patients is very low. Patients should avoid eating animal-sourced proteins as it produces acidity and favour baseproducing plant-sourced constituents like fruits and vegetables (Clinical pharmacy and therapeutics, 2012).

\section{Sodium management}

Sodium $\left(\mathrm{Na}_{+}\right)$is a mineral present in almost all-natural foods. Salt, on the other hand, is a sodium chloride compound. One of the three principal electrolytes in the body is sodium; the other two are potassium and chloride. Too much salt can add risk to renal disease because the kidneys are unable to remove enough sodium and water from the body. As the $\mathrm{Na}+$ and fluid accumulate in the tissues and bloodstream, they can lead to a variety of problems such as limbs and face oedema, increased thirst, elevated BP, and heart failure (due to extra fluid in the blood circulation forcing the heart to work too hard and causing it to expand and weaken). It can also cause shortness of breath owing to fluid buildup in the lungs, making breathing difficult. Salt or $\mathrm{Na}_{+}$in other forms may be present in the foods 
we eat. Processed foods usually have a higher level of sodium because they contain salt. Patients should track their sodium consumption by reading the food labels if any sodium is listed, compare brands, and use products with fewer sodium and spices not listed as "salt" in the name of the product, such as garlic powder rather than garlic salt. Patients should also prefer fresh over packaged meats, choose fresh vegetables and fruits, and avoid salted, canned, frozen, and processed foods. When preparing food, patients should avoid using salt and limit overall sodium intake to $400 \mathrm{mg}$ per meal and 150 mg per snack (National Kidney Foundation, 2014).

\section{Potassium management}

Potassium helps maintain the heartbeat regular and the muscles functioning properly. Potassium is also required to keep fluid and electrolyte balance in the bloodstream. In CKD patients, potassium intake should be monitored because when the kidneys are impaired, they can no longer eliminate enough potassium, resulting in a buildup of potassium in the blood. This condition, termed hyperkalemia, can cause muscle weakness, irregular heartbeats, slow pulse, heart attacks, and ultimately, death. Patients should lower the potassium in their diet by eating smaller portions of foods with higher protein content like meat, poultry, fish, beans, dairy, and nuts. Potassium chloride is used as a salt alternative in several packaged goods, such as canned soups and tomato products, and CKD patients should avoid it. Some of the foods containing less potassium are white rice, white bread, pasta, cooked rice, white cereals, and rice milk. Foods that contain more potassium are brown and wild rice, whole wheat bread and pasta, bran cereals, and cow's milk (Potassium Tips for People with Chronic Kidney Disease, 2011). Patients should also minimise potassium intake by eating low-potassium fruits and vegetables (American Kidney Fund, 2021). Fruits with less potassium are apples, apricots, berries, cranberry juice, grapefruit juice, lemons, limes, papayas, pears, peaches, plums, pineapples, and watermelon. Vegetables with less potassium are cabbage, onions, cauliflower, carrots, brinjal, summer squash, mushrooms, cucumber, apricots, and lettuce (Potassium Tips for People with Chronic Kidney Disease, 2011).

\section{Corrected Calcium}

The total calcium present in the blood is available in three forms: $40 \%$ of calcium is bound to proteins, $10 \%$ of calcium is complexed with anions like bicarbonates, lactate, and phosphates, and the remaining $50 \%$ of calcium is in the ionised form. From the total calcium of the blood, only ionised calcium is physiologically active.
The majority of the protein-bound calcium is bound to albumin. Moreover, any fluctuation in serum albumin levels will impact the total calcium concentration. Hence, serum albumin concentration should always be included when interpreting total calcium. A new formula has been developed to calculate "corrected" total calcium concentration from total calcium and serum albumin concentrations to reduce the influence of aberrant protein concentration on total calcium measurement. The amount of "corrected" calcium reflects ionised calcium precisely than the amount of uncorrected calcium in abnormal serum protein patients. Corrected total calcium levels in the blood should be kept within the laboratory's normal range, ideally at the lower end ( 8.4 to $9.5 \mathrm{mg} / \mathrm{dL}$ ) (Acute care testing org your scientific knowledge site, 2007).

Corrected calcium $(\mathrm{mg} / \mathrm{dL})=$ Measured total calcium
$(\mathrm{mg} / \mathrm{dL})+0.8(4-$ serum Albumin $\mathrm{g} / \mathrm{dL})$

\section{eGFR and its significance}

eGFR is the best test to determine renal function. It helps identify the stage of kidney disease and treat the kidney disease accordingly. eGFR can be calculated from serum creatinine, age, body weight, and gender. People with healthy kidneys have an eGFR higher than $90 \mathrm{~mL} / \mathrm{min} / \mathrm{m}^{2}$. The eGFR tells the stage of kidney disease and helps healthcare professionals plan the treatment. Generally, the Cockcroft-Gault formula is used to estimate eGFR, but some studies reported an overestimate or underestimate of the GFR. The CKD-EPI (Chronic Kidney Disease Epidemiology Collaboration) equation was developed in an attempt to formulate a more accurate formula for eGFR based on serum creatinine and other clinical data (Levey et al., 2009).

\section{MRPs in CKD patients}

An MRP involves drug therapy that interferes (or can interfere) with the patient's desired outcome. They are categorised as underdose, overdose, untreated conditions, adverse drug reactions, drug-drug interactions, and non-adherence to medication that may lead to therapy failure (Mason, 2011). CKD patients are always required to take multiple medications to treat casual conditions like diabetes mellitus, hypertension, and progression of CKD. When renal function is impaired, additional medications are needed to manage CKD problems, including mineral and bone disorders, anaemia, hyperlipidaemia, and cardiovascular problems. An increase in medication usage will lead to more MRPs. Non-adherence to the medication is one of the MRPs may lead to an increase in morbidity, mortality, hospitalisation length, and decreased quality of life (QoL); it also leads to increased healthcare costs (Mason, 2011). 


\section{MTM services}

MTM will significantly impact health-related quality of life (HRQoL) by finding and resolving MRPs. It will show the impact on health-related costs. MTM has five core elements to address MRPs.

Medication treatment review (MTR) is the first core element. In MTR, the patient has to show all of his medications to the pharmacist, including prescription, non-prescription medications, herbal medicines, and any form of dietary supplements. The pharmacist will evaluate all medications comprehensively for MRP, including adherence, and collaborate with the physician to resolve any MRP (Medication Therapy Management in Pharmacy Practice, 2008).

The second core element in MTM is the personal medication record (PMR), a complete record of patient medicines, i.e. prescription, non-prescription, and other dietary supplements. Patients receiving MTM services will provide a complete description of their medicines that is PMR, ideally generated electronically but can be generated manually. Whichever way the pharmacist creates the PMR, patients should understand it easily. The generated PMR given to the patient should include patient name, date of birth, patient phone number, emergency contact information (name, relationship, phone number), physician (name and phone number), and pharmacist (name and phone number). Allergies (e.g. what are the allergies the patient has? Other MRPs (e.g. what medication caused the problem? What was the problem the patient had?). For each medication in the PMR, the information should include medication name, dose, indication, starting date, stopping date, and instructions like when to take it (Medication Therapy Management in Pharmacy Practice, 2008).

The third core element in MTM is the medicationrelated action plan (MAP). This document includes a list of medical problems that the patient can use as part of self-management to achieve specific health goals and track progress. It also includes information like patient name, physician (name and contact number), pharmacist (name and contact number), date of MAP creation, action steps for the patient (e.g. "What I need to do..."), notes for the patient (e.g. "What I did and when I did it..."), and appointment information for follow-up with the pharmacist, if applicable (Medication Therapy Management in Pharmacy Practice, 2008).

The fourth core element is Intervention and/or referral. Numerous studies have demonstrated that interventions provided by the pharmacist related to MRPs had a positive impact. The resolution of MRPs involves the teamwork of pharmacists, doctors, nurses, and other healthcare professionals. The pharmacist delivers consulting services and intervenes to resolve MRPs. When needed, the pharmacist refers the patient to a physician or another healthcare expert. The pharmacist may collaborate with physicians or other healthcare professionals to rectify potential MRPs or work directly with the patient. Appropriate information given by the pharmacist to the physician or other healthcare professionals is integral to the intervention component of the MTM service; it includes medication selection, ideas to address MRPs, and suggested followup.

Documentation is the final core element of the MTM service. It is essential and based on services and interventions performed by a pharmacist, appropriate for assessing patient progress and sufficient for billing purposes (Medication Therapy Management in Pharmacy Practice, 2008).

\section{Conclusion}

The clinical pharmacist-assisted collaborative MTM services may improve medication adherence, decrease healthcare costs, and improve CKD patients' HRQoL by addressing MRPs, importing the knowledge about medications, and providing education on the disease, drugs, and lifestyle modifications.

\section{References}

Acute care testing org your scientific knowledge site. (2007). Ionized calcium (online). Available at: https://acutecaretesting.org/-

/media/acutecaretesting/files/pdf/ionized-calcium.pdf

Al Raiisi F, Stewart D, Fernandez-Llimos F, Salgado TM, Mohamed MF, Cunningham S. (2019). Clinical pharmacy practice in the care of Chronic Kidney Disease patients: a systematic review. Int J Clin Pharm. 41(3): 630-666. https://doi.org/10.1007/s11096-019-00816-4

American Kidney Fund. (2021). Fluid and stages of kidney disease (online). Available at:

https://kitchen.kidneyfund.org/generalnutrients/fluid/\#: :text=When\%20you\%20have\%20kidney\% 20disease,kidneys\%20hydrated\%20and\%20working\%20well

Couser, W. G., Remuzzi, G., Mendis, S., \& Tonelli, M. (2011). The contribution of chronic kidney disease to the global burden of major non-communicable diseases. Kidney international, 80(12), 1258-1270.

https://doi.org/10.1038/ki.2011.368

GBD Chronic Kidney Disease Collaboration (2020). Global, regional, and national burden of chronic kidney disease, 1990-2017: a systematic analysis for the Global Burden of Disease Study 2017. The Lancet, 395(10225), 709-733. https://doi.org/10.1016/S0140-6736(19)32977-0 
Hassan, Y., Al-Ramahi, R. J., Aziz, N. A., \&Ghazali, R. (2009). Impact of a renal drug dosing service on dose adjustment in hospitalized patients with chronic kidney disease. The Annals of pharmacotherapy, 43(10), 1598-1605. https://doi.org/10.1345/aph.1M187

Indian journal of nephrology. (2016). Guidelines for vaccination in patients with chronic kidney disease (online). Available at: http://isn-india.org/images/Indian-Society-ofNephrology-Guidelines-for-vaccination-of-Chronic-KidneyDisease.pdf

James, P. A., Oparil, S., Carter, B. L., Cushman, W. C., Dennison-Himmelfarb, C., Handler, J., et al. (2014). 2014 evidence-based guideline for the management of high blood pressure in adults: report from the panel members appointed to the Eighth Joint National Committee (JNC 8). JAMA, 311(5), 507-520.

https://doi.org/10.1001/jama.2013.284427

Ko, G. J., Obi, Y., Tortorici, A. R., \&Kalantar-Zadeh, K. (2017). Dietary protein intake and chronic kidney disease. Current opinion in clinical nutrition and metabolic care, 20(1), 77-85. https://doi.org/10.1097/MCO.0000000000000342

Levey, A. S., Stevens, L. A., Schmid, C. H., Zhang, Y. L., Castro, A. F., 3rd, Feldman, H. I., Kusek, J. W., et al. (2009). A new equation to estimate glomerular filtration rate. Annals of internal medicine, 150(9), 604-612.

https://doi.org/10.7326/0003-4819-150-9-20090505000006

Lin, H. W., Lin, C. H., Chang, C. K., Chou, C. Y., Yu, I. W., Lin, C. C., et al. (2018). Economic outcomes of pharmacistphysician medication therapy management for polypharmacy elderly: A prospective, randomized, controlled trial. Journal of the Formosan Medical Association, 117(3), 235-243.

https://doi.org/10.1016/j.jfma.2017.04.017

Marriott, J., Cockwell, P., Stringer, S. (2012). Chronic kidney disease and end-stage renal disease. In: Walker. R. \& Whittlesea. C. (Eds), Clinical pharmacy and therapeutics (pp.272-294). China: Churchill livingstone Elsevier

Mason N. A. (2011). Polypharmacy and medication-related complications in the chronic kidney disease patient. Current opinion in nephrology and hypertension, 20(5), 492-497. https://doi.org/10.1097/MNH.0b013e328349c261

Mason, D.L., Assimon, M. M. (2013). Renal disorders. In: B. K. Alldredge\& R. L. Corelli (Eds.), Applied therapeutics the clinical use of drugs (pp. 764-796). Philadelphia: Wolters Kluwer| Lippincott Williams and Wilkins

Medication Therapy Management in Pharmacy Practice. (2008). Core Elements of an MTM Service Model (online). Available at:

https://pharmacistsprovidecare.com/sites/default/files/cor e_elements_of_an_mtm_practice.pdf

MEDSAFE New Zealand Medicines and Medical Devices Safety Authority. (2015). Nitrofurantoin - Not Suitable in Renal Impairment (online). Available at:

https://www.medsafe.govt.nz/profs/PUArticles/December2 015/Nitrofurantoin.htm

Michigan Medicine University of Michigan. (2019).

Management of Chronic Kidney Disease. Available at:
http://www.med.umich.edu/1info/FHP/practiceguides/kidn ey/CKD.pdf

Mimura, I., Tanaka, T., Nangaku, M. (2015). How the Target Hemoglobin of Renal Anemia Should be?. Nephron clinical practice, 131: 202-209. https://doi.org/10.1159/000440849

National Institute for Health and Care Excellence. (2020) Hyperphosphatemia in chronic kidney disease overview (online). Available at:

http://pathways.nice.org.uk/pathways/hyperphosphataemi a-in-chronic-kidney-disease

National institute of diabetes and digestive and kidney diseases. (2010) Phosphorus Tips for People with Chronic Kidney Disease (CKD) (online). Available at: https://www.niddk.nih.gov/-/media/Files/HealthInformation/Health-Professionals/KidneyDisease/PhosphorusTipsforPeopleCKD_EN.pdf

National institute of diabetes and digestive and kidney diseases. (2010). Protein Tips for People with Chronic Kidney Disease (CKD) (online). Available at: https://www.niddk.nih.gov/-/media/Files/HealthInformation/Health-Professionals/KidneyDisease/ProteinTipsforPeopleCKD_EN.pdf

National institute of diabetes and digestive and kidney diseases. (2011). Potassium Tips for People with Chronic Kidney Disease (CKD) (online). Available at: https://www.niddk.nih.gov/-/media/Files/HealthInformation/Health-Professionals/KidneyDisease/PotassiumTipsforPeopleCKD_EN.pdf

National Kidney Foundation. (2014) Nutrition and Chronic Kidney Disease (STAGE 1-4) (online). Available at: https://www.kidney.org/sites/default/files/11-500114_docsnutrikidfail_stage1-4.pdf

Ohnishi J, Miyake A, Kuwatsuka K, Onoue Y, Lee M, Koyama $T$, et al. (2011). Effect of pharmacist management on serum hemoglobin levels with renal anemia in hemodialysis outpatients. Biol Pharm Bull. 34(10): 1609-12. https://doi.org/10.1248/bpb.34.1609

Onder, A. M., Espinoza, V., Berho, M. E., Chandar, J., Zilleruelo, G., \& Abitbol, C. (2006). Acute renal failure due to phenazopyridine (Pyridium) overdose: case report and review of the literature. Pediatric nephrology (Berlin, Germany), 21(11), 1760-1764.

https://doi.org/10.1007/s00467-006-0196-1

Public law 108-173. (2003). Medicare prescription drug improvement and modernization act of 2003 (online). Available at: https://www.congress.gov/108/plaws/publ173/PLAW108publ173.pdf

Rogers, J., Leung, M., Beaulieu, M., Levin, A., Burnett, S., \& Zienkiewicz, A. (2011). Evaluation of anemia management by algorithms in patients with chronic kidney disease who are not receiving dialysis. The Canadian journal of hospital pharmacy, 64(2), 141-146. https://doi.org/10.4212/cjhp.v64i2.998

Singh, A. K., Farag, Y. M., Mittal, B. V., Subramanian, K. K., Reddy, S. R., Acharya, V. N., et al. (2013). Epidemiology and risk factors of chronic kidney disease in India - results from the SEEK (Screening and Early Evaluation of Kidney Disease) 
study. BMC nephrology, 14, 114.

https://doi.org/10.1186/1471-2369-14-114

Stemer, G., \& Lemmens-Gruber, R. (2011). Clinical pharmacy activities in chronic kidney disease and end-stage renal disease patients: a systematic literature review. $B M C$ nephrology, 12, 35. https://doi.org/10.1186/1471-2369-1235

Wojcicki J. M. (2013). Hyperphosphatemia is associated with anemia in adults without chronic kidney disease: results from the National Health and Nutrition Examination Survey (NHANES): 2005-2010. BMC nephrology, 14, 178.

https://doi.org/10.1186/1471-2369-14-178 